\title{
Outcomes of Triple-Combination Therapy for Chronic Tinnitus
}

\author{
YING-HSU JUAN ${ }^{1,2}$, CHIU-TIEN HSU ${ }^{3}$ and JUEN-HAUR HWANG ${ }^{4,5,6}$ \\ ${ }^{1}$ Department of Traditional Chinese Medicine, Dalin Tzu Chi Hospital, \\ Buddhist Tzu Chi Medical Foundation, Chiayi, Taiwan, R.O.C.; \\ ${ }^{2}$ Graduate Institute of Integrated Medicine, College of Chinese Medicine, \\ China Medical University, Taichung, Taiwan, R.O.C.; \\ ${ }^{3}$ Department of Clinical Psychology, Dalin Tzu Chi Hospital, \\ Buddhist Tzu Chi Medical Foundation, Chiayi, Taiwan, R.O.C.; \\ ${ }^{4}$ Department of Otolaryngology-Head and Neck Surgery, Dalin Tzu Chi Hospital, \\ Buddhist Tzu Chi Medical Foundation, Chiayi, Taiwan, R.O.C.; \\ ${ }^{5}$ School of Medicine, Tzu Chi University, Hualien, Taiwan, R.O.C.; \\ ${ }^{6}$ Department of Medical Research, China Medical University Hospital, \\ China Medical University, Taichung, Taiwan, R.O.C.
}

\begin{abstract}
Background/Aim: Patients with tinnitus suffer not only from auditory dysfunction but also physical, mental, and social difficulties. Our aim was to investigate the outcomes of our triple-combination therapy for chronic tinnitus, which includes narrow-band noise masking, auricular acupuncture and cognitive behavioral therapy. Patients and Methods: The tinnitus handicap inventory (THI) scores of 78 adults with chronic tinnitus who received triple-combination therapy for 12 weeks were analyzed retrospectively. Results: There were 39 females and 39 males in this study. The mean age was 60.5 years [standard deviation $(S D)=13.0$, range $=27-88$ years] for all subjects. Mean pure tone average $=39.5$ decibel hearing level $(S D=23.09)$. Pre-treatment mean THI score was $41.4(S D=21.13)$, functional $(F)$ subscore was 17.9 $(S D=10.69)$, emotional $(E)$ subscore was $10.6(S D=6.87)$, and catastrophic $(C)$ subscore was $12.9(S D=4.85)$. Posttreatment THI total score was significantly lower compared to pre-treatment THI total score. Also, post-treatment subscores were significantly lower compared to pre-treatment subscores in $F$ and $C$ subsocres, but not in $E$ subscore. In addition, multivariate logistic regression analysis showed that
\end{abstract}

This article is freely accessible online.

Correspondence to: Juen-Haur Hwang, Department of Otolaryngology-Head and Neck Surgery, Dalin Tzu Chi Hospital, Buddhist Tzu Chi Medical Foundation, No. 2 Minsheng Road, Dalin, Chiayi, 62247, Taiwan, R.O.C. Tel: +886 52648000, Fax: +886 52648006, E-mail: g120796@ tzuchi.com.tw

Key Words: Chronic tinnitus, combined therapy, narrow-band noise, acupuncture, cognitive behavioral therapy, coffee, tea. coffee and tea consumption was significantly positively associated with a reduction of THI total score. Conclusion: Triple-combination therapy could be helpful for chronic tinnitus patients. It seemed that coffee and tea consumption may elicit increased improvement in conjunction with combined therapy for chronic tinnitus.

Tinnitus is a phantom sensation of non-speech sound in the absence of acoustic stimulation. Dysfunction in the cochlea, auditory and non-auditory cortices have been reported to be associated with auditory tinnitus (1). Patients with tinnitus suffer from not only auditory dysfunction, but also physical, mental, and social difficulties. Patients with tinnitus also often have symptoms consistent with vagal withdrawal and sympathetic overactivity (2). Many factors have been reported to be related with the occurrence and/or severity of auditory tinnitus. For example, age, gender, hearing loss and tinnitus duration were associated with tinnitus-related loudness and/or distress $(3,4)$. Increased caffeine intake has been demonstrated to be associated with a lower risk of tinnitus in women (5).

Any individual treatment for this complex disease may not achieve a satisfactory result. Thus, combined therapy, including tinnitus retraining therapy (TRT), sound therapy, acupuncture and/or psychotherapy, are applied in patients with chronic tinnitus $(2,6-8)$. TRT, which includes directive counseling and sound therapy, is used by patients with chronic tinnitus with success (8). It has been suggested that sound therapy could mask symptoms associated with tinnitus, acupuncture may improve the autonomic nervous dysfunction of tinnitus patients, and psychotherapy could relieve tinnitus-related emotional distress $(2,6-8)$.

In this study we aimed to investigate the outcomes of our ttriple-combination therapy (narrow-band noise masking, 
auricular acupuncture and cognitive behavioral therapy) in adult patients with chronic tinnitus.

\section{Patients and Methods}

Clinical data of 78 adult patients with chronic auditory tinnitus (duration $>6$ months) receiving combined tinnitus therapy between 2013 and 2016 at Dalin Tzu Chi Hospital were retrospectively investigated. The study was conducted in accordance with the Declaration of Helsinki (9) and was approved by The Research Ethics Committee of Dalin Tzu Chi Hospital, Buddhist Tzu Chi Medical Foundation (No. B10503006). Informed written consent was waived because the study was a retrospective data analysis.

The triple-combination therapy included: i) narrowed-band noise masking, ii) acupuncture, and iii) cognitive behavioral therapy. The narrowed-band noise was generated by a sound generator, depending on the frequency of each patient's tinnitus, which was determined by frequency matching. The narrowed-band noise with the loudness of a minimal masking level was given binaurally via a headset for 15 minutes. Auricular acupuncture was applied at three points: i) SJ 21, ii) SI 19, and iii) GB2, preauricularly (Figure 1) for 20 minutes by a traditional Chinese medicine doctor. SJ2 1 (Ermen) was indicated for tinnitus, deafness, otitis media, and toothache (10), SI19 (Tinggong) was indicated for tinnitus, deafness, otorrhea, toothache, and dysfunction of the temporomandibular joint (11), and GB2 (Tinghui) was indicated for tinnitus, deafness, and toothache (12). The cognitive behavioral therapy included an introduction to tinnitus and its related distress, as well as relaxation training, auto suggestion, and mindfulness. This was conducted as part of a group therapy for 30 minutes by a clinical psychotherapist.

Each of the three individual therapies was administered on the same day once per week for 12 weeks. Before therapy was administered, a questionnaire to collect clinical data, habits and pure tone audiometry was acquired. Severity of tinnitus was evaluated using the Tinnitus Handicap Inventory (THI) (13) before and immediately after therapy was administered. Taken together, there were 25 questions with three possible responses: i) yes, ii) sometimes, iii) no. Answers were scored as follows: i) 4 points for an answer of "yes", ii) 2 for an answer of "sometimes", and iii) 0 for an answer of "no". Thus, the total scores of the THI ranged between 0 and 100 (13). The higher the THI score were, the higher severity of tinnitus. The reduction of THI score was defined and calculated from pre-treatment data minus post-treatment data for each patient.

Exclusion criteria included: i) subjects aged younger than 20 years, ii) with external or middle ear disease, iii) conductive hearing loss (presenting with an air-bone gap on audiogram), iv) arteriovascular malformation, v) brain tumor or vestibular schwannoma, cancer, vi) Parkinson's disease, vii) dementia, viii) liver cirrhosis, ix) stroke, or $\mathrm{x}$ ) head and neck radiation exposure.

Statistical analysis. In this study, sex, hypertension, diabetes mellitus, dyslipidemia, coronary artery disease (CAD), chronic kidney disease (CKD), insomnia, smoking, alcohol drinking, coffee, tea, and high noise exposure were treated as the category variables. Furthermore, we also categorized the habits of coffee and/or tea consumption into four groups: i) without coffee and tea, ii) with coffee, iii) with tea, and iv) with coffee and tea. Age, body mass index (BMI), and pure tone average (PTA) were treated as the numerical variables. Student's t-test was used to test the differences of THI total score and subscores before and following therapy. Univariate linear regression analysis was used to test for the initial effects of individual variables on the reduction of THI total score. Multivariate logistic regression analysis was used to test the odds of prominent clinical factors on the reduction of THI total score $(\geq 10)$, with adjustment variables of $p$-Value $<0.2$ from the univariate linear regression analysis. All analyses were performed using STATA 10.0 software (Stata Corp, College Station, TX, USA).

\section{Results}

Subjects' clinical characteristics are shown in Table I. There was a total of 78 subjects (39 women and 39 men) included in the study. The mean subject age was 60.5 years $(\mathrm{SD}=13.0$; range: $27-88$ years old) for all subjects, 58.6 years $(\mathrm{SD}=13.0)$ for women and 62.4 years $(S D=12.9)$ for men. The mean body mass index (BMI) was $23.2 \mathrm{~kg} / \mathrm{m}^{2}(\mathrm{SD}=3.0)$ for all subjects, $22.6 \mathrm{~kg} / \mathrm{m}^{2}(\mathrm{SD}=3.2)$ for women, and $23.9 \mathrm{~kg} / \mathrm{m}^{2}$ $(\mathrm{SD}=2.6)$ for men. The mean pure tone threshold on audiometry (PTA) was 39.5 decibel hearing level (dBHL) $(\mathrm{SD}=23.1)$ for all subjects, $36.3 \mathrm{dBHL}(\mathrm{SD}=24.8)$ for women, and $42.7 \mathrm{dBHL}(\mathrm{SD}=21.1)$ for men.

Figure 2 shows comparisons of the THI scores before and after treatment. The mean pre-treatment THI total score was $41.4(\mathrm{SD}=21.1)$, the functional $(\mathrm{F})$ subscore was 17.9 $(\mathrm{SD}=10.7)$, the emotional $(\mathrm{E})$ subscore was $10.6(\mathrm{SD}=6.9)$, and the catastrophic $(\mathrm{C})$ subscore was $12.9(\mathrm{SD}=4.9)$. The mean post-treatment THI total score was $30.9(\mathrm{SD}=17.0)$, the F subscore was $13.6(\mathrm{SD}=8.1)$, the $\mathrm{E}$ subscore was 9.6 $(\mathrm{SD}=6.5)$, and the $\mathrm{C}$ subscore was $7.7(\mathrm{SD}=4.1)$. Following treatment, the mean reduction of the THI score was 10.5 $(\mathrm{SD}=21.0)$, the $\mathrm{F}$ subscore was $4.3(\mathrm{SD}=10.5)$, the $\mathrm{E}$ subscore was $1.0(\mathrm{SD}=6.8)$, and the $\mathrm{C}$ subscore was $5.2(\mathrm{SD}=5.1)$.

The post-treatment THI total score was significantly lower compared to the pre-treatment THI total score (Student's $t$-test, $p<0.0001)$. Also, post-treatment subscores were significantly lower compared to pre-treatment subscores in F (Student's $t$ test, $p=0.0005$ ) and $\mathrm{C}$ (Student's $t$-test, $p<0.0001)$ subsocres, but not in E subscore (Student's $t$-test, $p=0.1996$ ).

Table II shows the effects of individual variables on the THI total score by univariate linear regression analysis. Among all the tested variables, only hypertension and dyslipidemia were significantly positively associated with the pre-treatment THI total score. Otherwise, coffee and tea consumption was significantly positively associated with the reduction in the THI total score.

Multivariate logistic regression analysis on the significant reduction $(\geq 10)$ of the THI total score by prominent clinical factors is shown in Table III. Coffee and tea consumption [odds ratio (OR) $\pm \mathrm{SE}=2.01 \pm 0.47, p=0.003$ ], but not sex $(\mathrm{OR} \pm \mathrm{SE}=0.54 \pm 0.28, p=0.255)$ or dyslipidemia $(\mathrm{OR} \pm \mathrm{SE}=$ $1.27 \pm 0.87, p=0.732$ ), was significantly positively associated with a significant reduction in the THI total score. 


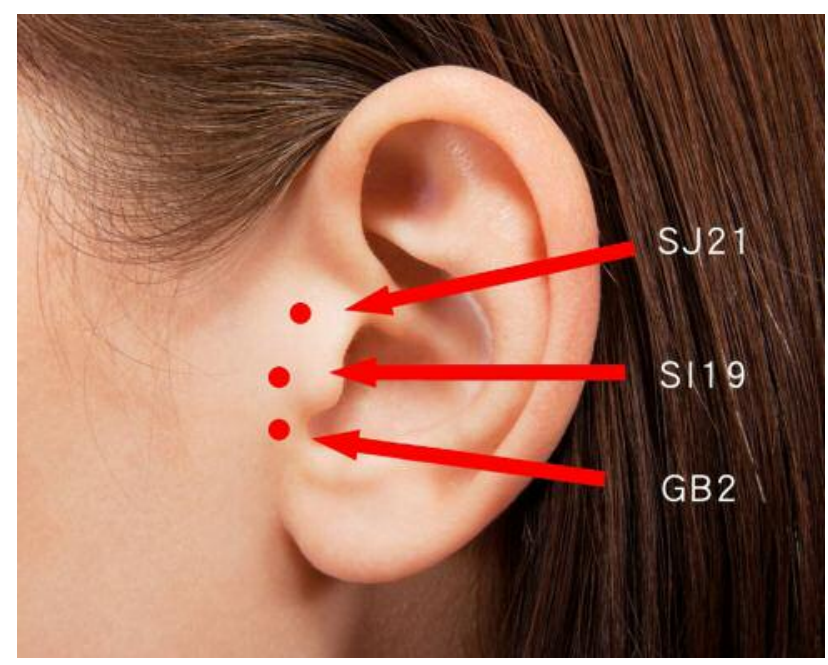

Figure 1. Auricular acupuncture sites. Acupuncture was applied at three points: i) SJ 21, ii) SI 19, and iii) GB2 preauricularly. Informed consent was obtained from the patient for the publication of this image.

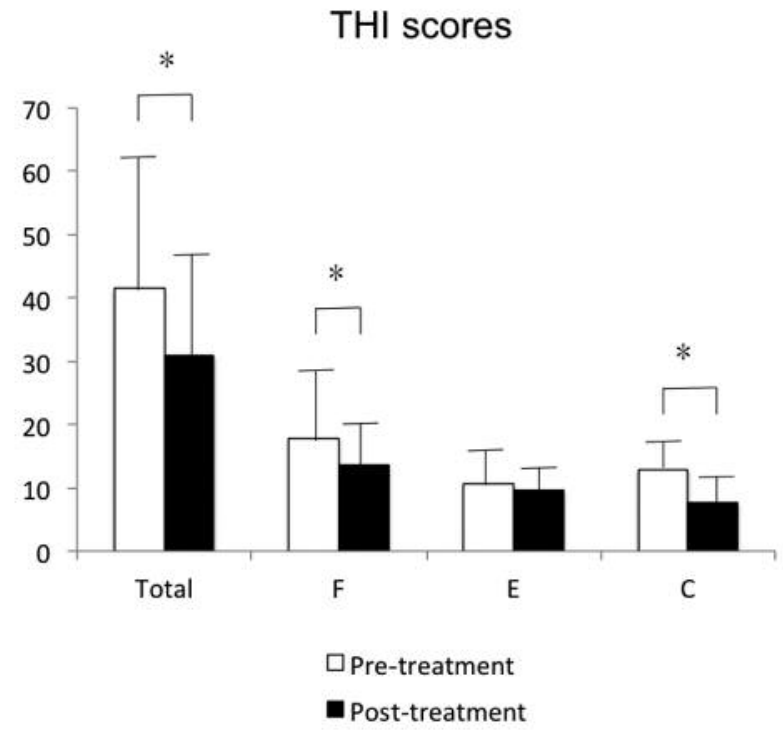

Figure 2. Comparisons of THI scores before and after treatment. Posttreatment THI total score was significantly lower compared to pretreatment THI total score (Student's t-test, $p<0.0001$ ). Also, posttreatment subscores were significantly lower compared to pre-treatment subscores in $F$ (Student's t-test, $p=0.0005$ ) and $C$ (Student's t-test, $p<0.0001)$ subsocres, but not to E subscore (Student's $t$-test, $p=0.1996$ ).

\section{Discussion}

This retrospective study showed that our triple-combination therapy of narrow-band noise masking, auricular acupuncture and cognitive behavioral therapy for 12 weeks, could reduce the severity of chronic tinnitus in the Taiwanese population.
Table I. Baseline patient characteristics $(N=78)$.

\begin{tabular}{|c|c|}
\hline Variables & Values \\
\hline Age $($ mean $\pm S D, y r)$ & $60.5 \pm 13.0$ \\
\hline Gender (n, M/F) & $39 / 39$ \\
\hline $\mathrm{BMI}\left(\mathrm{mean} \pm \mathrm{SD}, \mathrm{kg} / \mathrm{m}^{2}\right)$ & $23.3 \pm 3.0$ \\
\hline $\mathrm{PTA}($ mean $\pm \mathrm{SD}, \mathrm{dBHL})$ & $39.5 \pm 23.1$ \\
\hline Pre-treatment THI total score $($ mean $\pm S D)$ & $41.4 \pm 21.1$ \\
\hline Pre-treatment $\mathrm{F}$ subscore $($ mean $\pm \mathrm{SD})$ & $17.9 \pm 10.7$ \\
\hline Pre-treatment $\mathrm{E}$ subscore $($ mean $\pm \mathrm{SD})$ & $10.6 \pm 6.9$ \\
\hline Pre-treatment $\mathrm{C}$ subscore $($ mean $\pm \mathrm{SD})$ & $12.9 \pm 4.9$ \\
\hline Post-treatment THI total score $($ mean \pm SD) & $30.9 \pm 17.0$ \\
\hline Post-treatment $\mathrm{F}$ subscore $($ mean $\pm \mathrm{SD})$ & $13.6 \pm 8.1$ \\
\hline Post-treatment $E$ subscore (mean \pm SD) & $9.6 \pm 6.5$ \\
\hline Post-treatment $\mathrm{C}$ subscore $($ mean $\pm \mathrm{SD})$ & $7.7 \pm 4.1$ \\
\hline Reduction of THI total score (mean \pm SD) & $10.5 \pm 21.0$ \\
\hline Reduction of F subscore $($ mean \pm SD) & $4.3 \pm 10.5$ \\
\hline Reduction of E subscore (mean \pm SD) & $1.0 \pm 6.8$ \\
\hline Reduction of C subscore $($ mean $\pm \mathrm{SD})$ & $5.2 \pm 5.1$ \\
\hline Hypertension (\%) & 18.0 \\
\hline Diabetes mellitus (\%) & 9.0 \\
\hline Dyslipidemia (\%) & 14.1 \\
\hline Coronary artery disease $(\%)$ & 11.5 \\
\hline Chronic kidney disease $(\%)$ & 5.1 \\
\hline Insomnia $(\%)$ & 41.0 \\
\hline Smoking (\%) & 6.4 \\
\hline Alcohol drinking (\%) & 23.1 \\
\hline Coffee $(\%)$ & 52.6 \\
\hline Tea $(\%)$ & 70.5 \\
\hline High noise exposure $(\%)$ & 46.8 \\
\hline
\end{tabular}

BMI: Body mass index; dBHL: decibel hearing level; F: female; M: male; PTA: pure tone threshold on audiometry; SD: standard deviation; THI: tinnitus handicap inventory.

Although a previous study has shown that caffeine could reduce the risk of tinnitus in women (5), we found that only hypertension and dyslipidemia, but not coffee or tea consumption, were positively related with the severity of chronic tinnitus prior to treatment. Furthermore, subjects who consumed coffee or tea, compared to subjects without these two habits, showed greater improvement following the combined treatment for chronic tinnitus.

Tinnitus is a complex disease with multiple adverse effects for patients, not only concerning the accompanying sound but also the related psychological disturbances (2). Several studies have shown that the severity and/or risk of tinnitus would be related with some clinical factors. For example, loudness and annoyance of tinnitus were generally higher in older subjects, those with binaural or centrally perceived tinnitus, those with persistent tinnitus, and those who had coexisting hearing loss, vertigo, and hyperacusis (14). Annoying tinnitus was also increased in patients with a history of smoking, those reporting less sleep, those with increased stress levels, those in smaller households, those with a history of hypertension, hyperlipidemia, osteoarthritis, 
Table II. Effects of individual variables on the THI total score (univariate linear regression analysis). The values in each cell are shown as coefficients \pm standard errors with $p$-Value in the parentheses.

\begin{tabular}{lrrr}
\hline Variables & Before treatment & After treatment & Reduction \\
\hline Age & $-0.17 \pm 0.19(0.380)$ & $0.04 \pm 0.15(0.787)$ & $-0.21 \pm 0.19(0.268)$ \\
Gender (M versus F) & $-6.85 \pm 4.75(0.154)$ & $2.00 \pm 3.88(0.607)$ & $-8.85 \pm 4.68(0.062)$ \\
BMI & $0.07 \pm 0.85(0.934)$ & $-0.04 \pm 0.69(0.955)$ & $0.11 \pm 0.87(0.900)$ \\
PTA & $0.18 \pm 0.10(0.085)$ & $0.19 \pm 0.09(0.029)$ & $-0.01 \pm 0.10(0.907)$ \\
Hypertension & $18.84 \pm 5.89(0.002)$ & $13.39 \pm 4.82(0.007)$ & $5.45 \pm 6.21(0.382)$ \\
Diabetes mellitus & $-6.09 \pm 8.40(0.471)$ & $0.93 \pm 6.79(0.892)$ & $-7.01 \pm 8.33(0.403)$ \\
Dyslipidemia & $13.82 \pm 6.74(0.044)$ & $4.59 \pm 5.55(0.411)$ & $9.23 \pm 6.79(0.178)$ \\
CAD & $-0.07 \pm 7.54(0.992)$ & $7.93 \pm 6.01(0.191)$ & $-8.00 \pm 7.43(0.285)$ \\
CKD & $-2.26 \pm 10.92(0.836)$ & $-9.88 \pm 8.73(0.261)$ & $7.61 \pm 10.82(0.484)$ \\
Insomia & $7.27 \pm 4.83(0.136)$ & $1.28 \pm 3.94(0.747)$ & $6.00 \pm 4.82(0.217)$ \\
Smoking & $-6.41 \pm 9.81(0.515)$ & $-13.11 \pm 7.78(0.096)$ & $6.70 \pm 9.74(0.493)$ \\
Alcohol drinking & $-2.54 \pm 7.51(0.658)$ & $-2.00 \pm 4.60(0.665)$ & $-0.54 \pm 5.68(0.925)$ \\
Coffee & $0.19 \pm 4.82(0.969)$ & $-9.70 \pm 3.73(0.011)$ & $9.90 \pm 4.66(0.037)$ \\
Tea & $6.79 \pm 5.22(0.198)$ & $-4.13 \pm 4.23(0.332)$ & $10.92 \pm 5.10(0.035)$ \\
High noise exposure & $1.84 \pm 4.88(0.708)$ & $6.15 \pm 3.87(0.116)$ & $-4.31 \pm 4.83(0.375)$ \\
\hline
\end{tabular}

rheumatoid arthritis, asthma, depression, thyroid disease, an abnormal tympanic membrane, and increased noise exposure (3, 15-18). Hyperinsulinemia $(17,19)$, drug abuse in boys and exposure to second hand smoke in girls (20), obesity, and diabetes mellitus (17), have also been identified as risk factors of tinnitus. However, the role of the sex difference on tinnitus loudness and annoyance is still inconclusive (14, 15). Also, higher caffeine intake may reduce the risk of tinnitus in women (5). On the contrary, the acute effects of caffeine withdrawal may add to the burden associated with tinnitus (21).

In the aspect of tinnitus treatment, various sound therapies have demonstrated significant benefit for patients with tinnitus $(6,7)$. Some authors have reported that acupuncture alone (22) or combined with Qi-training (2) could modulate cardiac autonomic functions and reduce tinnitus-related symptoms in patients with chronic tinnitus. However, a meta-analysis has concluded that the beneficial effect of traditional Chinese acupuncture remains inconclusive for patients with tinnitus $(23,24)$. In this study, we found that the $\mathrm{F}$ and $\mathrm{C}$ subscores, but not the $\mathrm{E}$ subscore, were significantly decreased following our triple-combination therapy. These findings raised an interesting issue around sound therapy, acupuncture, and especially cognitive behavioral therapy, which could not relieve tinnitus-related emotional distress effectively. We suggest that other types of psychologic therapy should be employed in the future to help reduce tinnitus-related emotional problems.

The factors that affect the outcomes associated with chronic tinnitus treatment are poorly reported in the literature. For example, prognosis of sudden sensorineural hearing loss, due to tinnitus, is poor in cases of severe
Table III. The effects of coffee and tea, and prominent variables on significant reduction $(\geq 10)$ of THI scores (multivariate logistic regression analysis). * "Coffee and Tea" was treated as a category variable with four groups, as mentioned in our method.

\begin{tabular}{lccc}
\hline Variables & Odds ratio & Standard error & $p$-Value \\
\hline Sex (M versus $\mathrm{F})$ & 0.54 & 0.28 & 0.255 \\
Coffee and Tea* & 2.01 & 0.47 & 0.003 \\
Dyslipidemia & 1.27 & 0.87 & 0.732 \\
\hline
\end{tabular}

Pseudo R square $=0.1026$.

hearing loss or long-lasting tinnitus (25). Pre-treatment tinnitus severity has been negatively associated with the TRT outcome $(26,27)$. Baseline tinnitus severity and duration have also been found to influence repetitive transcranial magnetic stimulation (rTMS) outcomes (28). On the other hand, higher levels of education have been reported to be associated with increased improvement in a habituationbased training for patients with tinnitus (29). A diabetic diet might result in a significant improvement in complaints associated with tinnitus in patients with hyperinsulinemia (19). Improvement in the THI score have been positively correlated with pre-treatment activities of the left insula and the left rostral and pregenual anterior cingulate cortices, which control parasympathetic activity (30). However, improvement in loudness was negatively correlated with the activities of the right auditory cortices and parahippocampus (30). In the current study, we found that coffee and tea consumption was positively associated with a good prognosis of the tinnitus treatment. 
As previously reported, neural inflammation and/or damage resulting from oxidative stress might represent novel mechanisms of tinnitus (31-35). Furthermore, caffeine has been demonstrated to have an anti-oxidative effect, providing possible benefits to patients with tinnitus (36), despite its possible involvement with the increase in sympathetic tone (37). Taken together, previously published reports $(18,21)$, suggest that caffeine-containing coffee and tea may be beneficial for the prevention of tinnitus and as part of a treatment regimen. These results might also encourage patients with tinnitus to maintain their consumption of coffee and/or tea during their treatment, with no need to withdraw, as Claire et al., have previously suggested (21).

Herein we report that our triple-combination therapy was helpful for our patients with chronic tinnitus. Patients who consumed coffee and tea had improved outcomes during the therapy, and thus they should be encouraged to maintain this habit. These findings provide important insight and nutritional options for supplementing tinnitus treatment. However, additional large-scale, prospective, clinical trials should be conducted in order to provide additional and more convincing data in the future.

\section{Conflicts of Interest}

The Authors declare no conflicts of interest.

\section{Authors' Contributions}

YHJ performed the treatment and wrote the manuscript. CTH performed the treatment. JHH designed the study, performed the treatment, analyzed data, wrote manuscript and supervised the study.

\section{References}

1 Issa M, Bisconti S, Kovelman I, Kileny P and Basura GJ: Human auditory and adjacent non auditory cerebral cortices are hypermetabolic in tinnitus as measured by functional nearinfrared spectroscopy (fNIRS). Neural Plast 2016: 7453149, 2016. PMID: 27042360. DOI: 10.1155/2016/7453149

2 Choi EJ, Yun Y, Yoo S, Kim KS, Park JS and Choi I: Autonomic conditions in tinnitus and implications for Korean medicine. Evid Based Complement Alternat Med 2013: 402585, 2013. PMID: 24023574. DOI: 10.1155/2013/402585

3 Scott B, Lindberg P, Melin L and Lyttkens L: Predictors of tinnitus discomfort adaptation and subjective loudness. Br J Audiol 24(1): 51-62, 1990. PMID: 2317601.

4 Seydel C, Haupt H, Olze H, Szczepek AJ and Mazurek B: Gender and chronic tinnitus: differences in tinnitus-related distress depend on age and duration of tinnitus. Ear Hear 34(5): 661-672, 2013. PMID: 23439056. DOI: 10.1097/AUD.0b0 $13 \mathrm{e} 31828149 \mathrm{f} 2$

5 Glicksman JT, Curhan SG and Curhan GC: A prospective study of caffeine intake and risk of incident tinnitus. Am J Med 127(8): 739-743, 2014. PMID: 24608016. DOI: 10.1016/ j.amjmed.2014.02.033
6 Liu Z, Liang Y, Yang C, Liu Y, Li Y, Han X, Zeng L and He P: The clinical efficacy of composite acoustic therapy in patients of sudden deafness with tinnitus. Lin Chung Er Bi Yan Hou Tou Jung Wai Ke Za Zhi 29(4): 330-333. 2015. PMID: 26121832.

7 Stein A, Wunderlich R, Lau P, Engell A, Wollbrink A, Shaykevich A, Kuhn JT, Holling H, Rudack C and Pantev C: Clinical trial on tonal tinnitus with tailor-made notched music training. BMC Neurol 16: 38, 2016. PMID: 26987755. DOI: 10.1186/s12883-016-0558-7

8 Jastreboff PJ: 25 years of tinnitus retraining therapy. HNO 63(4): 307311, 2015. PMID: 25862626. DOI: 10.1007/s00106-014-2979-1

9 Declaration of Helsinki. World Medical Association. Available at: http://www.wma.net/e/ethicsunit/helsinki.htm [accessed on 24 June 2008].

$10 \mathrm{Xia} \mathrm{H}$ and Xiao S: The clinical observation of 30 tinnitus cases that treat with integrated traditional and western medicine. Acta Acad Med Jiangxi 44(1): 137, 2004.

11 Marks NJ, Emery P and Onisiphorou C: A controlled trial of acupuncture in tinnitus. J Laryngol Otol 98(11): 1103-1109, 1984. PMID: 6387018.

12 Jeon SW, Kim KS and Nam HJ: Long-term effect of acupuncture for treatment of tinnitus: a randomized, patient- and assessorblind, sham-acupuncture-controlled, pilot trial. J Altern Complement Med 18(7): 693-699, 2012. PMID: 22747248. DOI: 10.1089/acm.2011.0378

13 Kam AC, Cheung AP, Chan PY, Leung EK, Wong TK, van Hasselt $\mathrm{CA}$ and Tong MC: Psychometric properties of the Chinese (Cantonese) Tinnitus Handicap Inventory. Clin Otolaryngol 34: 309315, 2009. PMID: 19673977. DOI: 10.1111/j.1749-4486. 2009.01946.x

14 Hiller W and Goebel G: Factors influencing tinnitus loudness and annoyance. Arch Otolaryngol Head Neck Surg 132(12): 1323-1330, 2006. PMID: 17178943. DOI: 10.1001/archotol. 132.12 .1323

15 Kim HJ, Lee HJ, An SY, Sim S, Park B, Kim SW, Lee JS, Hong SK and Choi HG: Analysis of the prevalence and associated risk factors of tinnitus in adults. PLoS One 10(5): e0127578, 2015. PMID: 26020239. DOI: 10.1371/journal.pone.0127578

16 Miguel GS, Yaremchuk K, Roth T and Peterson E: The effect of insomnia on tinnitus. Ann Otol Rhinol laryngol 123(10): 696700, 2014. PMID: 24816421. DOI: 10.1177/0003489414532779

17 Kaźmierczak H and Doroszewska G: Metabolic disorders in vertigo, tinnitus, and hearing loss. Int Tinnitus J 7(1): 54-58, 2001. PMID: 14964957

18 Yang P, Ma W, Zheng Y, Yang H and Lin H: A systematic review and meta-analysis on the association between hypertension and tinnitus. Int J Hypertens 2015: 583493, 2015. PMID: 26881064. DOI: $10.1155 / 2015 / 583493$

19 Basut O, Ozdilek T, Coskun H, Erişen L, Tezel I, Onart S and Hizalan I: The incidence of hyperinsulinemia in patients with tinnitus and the effect of a diabetic diet on tinnitus. Kulak Burun Bogaz Ihtis Derg 10(5): 183-187, 2003. PMID: 12970590.

20 Marmut Z, Belojevic G, Backovic D, Zivojinovic JI, Tomanic M and Hadzic E: Tinnitus among Serbian secondary school students in relation to their Behavior and habits. Noise Health 16(69): 7378, 2014. PMID: 24804710. DOI: 10.4103/1463-1741.132080

21 Claire LS, Stothart G, McKenna L and Rogers PJ: Caffeine abstinence: an ineffective and potentially distressing tinnitus therapy. Int J Audiol 49(1): 24-29, 2010. PMID: 20053154. DOI: $10.3109 / 14992020903160884$ 
22 Laureano MR, Onishi ET, Bressan RA, Neto PB, Castiglioni ML, Batista IR, Reis MA, Garcia MV, de Andrade AN, Sanchez ML, Moreira HC, de Almeida RR, Garrido GJ and Jackowski AP: The effectiveness of acupuncture as a treatment for tinnitus: a randomized controlled trial using 99mTc-ECD SPECT. Eur Radiol 26(9): 3234-3242. 2016. PMID: 26747258. DOI: 10.1007/s00330-015-4164-7

23 Kim JI, Choi JY, Lee DH, Choi TY, Lee MS and Ernst E: Acupuncture for the treatment of tinnitus: a systematic review of randomized clinical trials. BMC Complement Altern Med 12: 97, 2012. PMID: 22805113. DOI: 10.1186/1472-6882-12-97

24 Liu F, Han X, Li Y and Yu S: Acupuncture in the treatment of tinnitus: a systematic review and meta-analysis. Eur Arch Otorhinolaryngol 273(2): 285-294, 2016. PMID: 25344063. DOI: $10.1007 / \mathrm{s} 00405-014-3341-7$

25 Mühlmeier G, Baguley D, Cox T, Suckfüll M and Meyer T: Characteristics and spontaneous recovery of tinnitus related to idiopathic sudden sensorineural hearing loss. Otol Neurotol 37(6): 634-341, 2016. PMID: 27228021. DOI: 10.1097/MAO.00 00000000001081

26 Koizumi T, Nishimura T, Sakaguchi T, Okamoto M and Hosoi $\mathrm{H}$ : Estimation of factors influencing the results of tinnitus retraining therapy. Acta Otolaryngol Suppl 562: 40-45, 2009. PMID: 19848238.

27 Molini E, Faralli M, Calzolaro L and Ricci G: Impact of identifying factors which trigger bothersome tinnitus on the treatment outcome in tinnitus retraining therapy. ORL J Otorhinolaryngol Relat Sepc 76(2): 81-88, 2014. PMID: 24777173. DOI: $10.1159 / 000360994$

28 Frank G, Kleinjung T, Landgrebe M, Vielsmeier V, Steffenhagen C, Burger J, Frank E, Vollberg G, Hajak G and Langguth B: Left temporal low-frequency rTMS for the treatment of tinnitus: clinical predictors of treatment outcome--a retrospective study. Eur J Neurol 17(7): 951-956, 2010. PMID: 20158510. DOI: 10.1111/j.1468-1331.2010.02956.x

29 Kröner-Herwig B, Zachriat C and Weigand D: Do patient characteristics predict outcome in the outpatient treatment of chronic tinnitus? Psychosoc Med 3: Doc07, 2006. PMID: 19742075
30 Kim SH, Jang JH, Lee SY, Han JJ, Koo JW, Vanneste S, De Ridder D and Song JJ: Neural substrates predicting short-term improvement of tinnitus loudness and distress after modified tinnitus retraining therapy. Sci Rep 6: 29140, 2016. PMID: 27381994. DOI: $10.1038 /$ srep29140

31 Hwang JH, Chang NC, Chen JC and Chan YC: Expression of antioxidant genes in the mouse cochlea and brain in salicylateinduced tinnitus and effect of treatment with spirulina platensis water extract. Audiol Neurotol 20(5): 322-329, 2015. PMID: 26277928. DOI: $10.1159 / 000381934$

32 Hwang JH, Chen JC and Chan YC: Effects of C-phycocyanin and Spirulina on salicylate-induced tinnitus, expression of NMDA receptor and inflammatory genes. PLoS One 8(3): e58215, 2013. PMID: 23533584. DOI: 10.1371/journal.pone. 0058215

33 Hwang JH, Chen JC, Yang SY, Wang MF and Chan YC: Expression of TNF-alpha and IL-1beta genes in the cochlea and midbrain in salicylate-induced tinnitus. J Neuroinflamm 8: 30 , 2011. PMID: 21477330. DOI: 10.1186/1742-2094-8-30

34 Hwang JH, Chen JC, Yang SY, Wang MF, Liu TC and Chan YC: Expression of COX- and NMDA receptor genes at the cochlea and midbrain in salicylate-induced tinnitus. Laryngoscope 121: 361-364, 2011. PMID: 21271589. DOI: 10.1002/lary.21283

35 Hwang JH, Huang Davis CW, Lu YC, Yang WS and Liu TC: Effects of tumor necrosis factor blocker on salicylate-induced tinnitus in mice. Int Tinnitus J 21(1): 23-28, 2017. PMID: 28723598. DOI: $10.5935 / 0946-5448.20170006$

36 Hwang JH, Chan YC, Hsu CJ, Liu TC and Chen JC: Effects of tea drinking on auditory functions in aged subjects. J Nutr Health Aging 16(3): 252-256, 2012. PMID: 22456782.

37 Mahmud A and Feely J: Acute effect of caffeine on arterial stiffness and aortic pressure waveform. Hypertension 38(2): 227231, 2001. PMID: 11509481. 